\title{
Gravitational effects on entangled states and interferometer with entangled atoms*
}

\author{
Horst von Borzeszkowski ${ }^{a \dagger}$ \\ Michael B. Mensky ${ }^{a b \ddagger}$ \\ ${ }^{a}$ Institut für Theoretische Physik, Technische Universität Berlin, \\ Hardenbergstr. 36, D-10623 Berlin, Germany \\ ${ }^{b}$ P.N.Lebedev Physical Institute, 53 Leninskii prosp., \\ 117924 Moscow, Russia
}

\begin{abstract}
It is discussed how systems of quantum-correlated (entangled) particles or atoms behave in external gravitational fields and what gravitational effects may exist in such systems. An experimental setup is proposed which improves the sensitivity of the Ramsey interferometer by the usage of quantum-correlated atoms. Entanglement of $n$ atoms improves the sensitivity to small phase shifts in $n^{2}$ times. This scheme may be used for observing gravity-induced phase shifts in laboratory.
\end{abstract}

\section{Entangled particles in gravitational fields}

Quantum-correlated, or entangled, states of two or more systems (for example particles or atoms) proved to be useful for constructing a new class of quantum devices for information transfer [1], quantum cryptography [2] and quantum computing [3]. We shall consider the possibility to use them for observing gravitational effects.

\footnotetext{
*Physics Letters A 286, 102-106 (2001)

†Email: borzeszk@itp.physik.tu-berlin.de

†Email: mensky@sci.lebedev.ru
} 


\subsection{Destruction of correlation by gravity}

It has been shown in the preceding paper 4 that correlation of the particles forming a EPR-pair may be violated if the particles propagate along different trajectories in a gravitational field. The violation may have the character of distortion (when the correlation still exists but is altered) or blurring (when the correlation is partly or completely washed out).

As an example a pair of spin-1/2 particles in correlated spin states (obtained in the course of the decay of a spin-0 particle) has been considered in [4]. As a result of the correlation, a measurement of the spin projection of one of the particles informs the experimenter about the spin projection of the other. In case of spinning particles having been obtained by a decay of a spinless particle, their spin projections on the same axis must be oppositely directed.

If external fields are absent, the spin correlation is maintained even if the particles move away from each other to any distance. However any field which rotate spins, among them gravitational field, destructs the correlation or blurs it. The effect is especially dramatic for gravitational field since the very concept of space direction (along which the spin projections have to be measured) cannot be defined globally in this case, but only in each point separately [4].

\subsection{Violation of spin correlation in gravitational field}

In principle this effect may be used for observing gravitational field. If for example all fields except for the gravitational field are removed (by screening) but nevertheless the experimenter observes that the spin correlation is violated, then the violation is an evidence of the presence of a gravitational field.

Correlation may exist and can be experimentally observed (or alternatively, its violation may be observed) even in a gravitational field where no global definition of space direction (no teleparallelism) exists. For this end repeated experiments are needed. Arranging the decay of a spin-0 particle many times and each time measuring the spin projections of the fragment particles on different axes, the experimenter may either find a pair of axes (in two different points) such that the corresponding spin projections are $100 \%$ correlated or prove that no such pair of axes exists. In the latter case the correlation will be proved to be blurred. In the first case it is only distorted. The reason for the distortion is a rotation of spins in the gravitational field. One may discover this effect by investigating the relation between the two 
axes (in spatially distant points) found in the series of correlation experiments (call them, for simplicity, the correlated axes). As a result of this investigation, a conclusion about existence and character of the gravitational field may be drawn.

Indeed, identifying spatial directions in neighboring points, an experimenter may, step by step, transfer these directions along any curve. This is possible, at least, if the gravitational field may be considered to be static. The result of such transfer may be mathematically described by the parallel transport of a local basis. Thus, two axes in spatially distant point may be put into correspondence by the parallel transfer along a certain curve. Let us apply this to the axes found in the correlation experiments ("correlated axes"). If the parallel transport along some curve (close to a geodesic) transfers one of the correlated axes into the other, then there is no evidence of a non-vanishing gravitational field. If the parallel transport transfers one of the correlated axes into the axis which differs from the second correlated axis by some rotation, then the conclusion is that a non-vanishing gravitational field is present which rotates the particles' spins.

We do not think that the effect of a gravitational field on the correlation of spins is directly observable by the present experimental technics, so that we do not elaborate the question in more detail. Note however that this effect may turn out to be important in some specific situations (for example as a part of more complicated phenomena in astrophysics) or can become significant due to future development of technology.

\subsection{Other gravitational effects based on entanglement}

The correlation of spins is not the only type of entanglement which may in principle lead to gravitational effects. Spins may be rotated by a gravitational field because and if the rotation group is a subgroup of the holonomy group of the given gravitational field. In generic case a holonomy group is a subgroup of the Lorentz group. Therefore it may include also boosts (transitions between reference frames moving with different velocities). Let two photons be prepared in some point in such a way that their frequencies are correlated. It may happen that each of the frequencies has an undetermined value but the frequencies of the two photons are correlated (in the sense of quantum correlation, i.e., by entanglement). For example, the energies

of the separate photons may be indefinite but the total energy is fixed. If then the photons are traveling into another point along different paths in a gravitational field, the correlation between their frequencies may be violated 
(distorted or blurred) when the photons arrive at the final point. This is nothing else than the effect of the gravitational red shift but now considered for a pair of entangled photons.

Moreover, in principle gravitational effects connected with the affine holonomy group may also be observed. This brings also the translation group into play, besides the Lorentz group. Therefore, gravitationally induced time and/or space shifts may be observed with the help of a pair of quantumcorrelated particles (or even with a greater number of correlated particles).

\section{Detection of gravity by an interferometer with entangled atoms}

Now let us consider ordinary schemes of observing gravitational effects which include no quantum correlation and show that even in such schemes the effect may be increased by quantum correlation. We shall present a scheme which makes use of quantum correlation to increase the gravity-induced phase shift. More precisely, it will be shown that the quantum correlation of two or more atoms may be used to add up the phase shifts of singular atoms in Ramsey interferometer.

\subsection{Idea of an interferometer with entanglement}

The idea is as follows (see Fig. 1). Owing to the action of an external, for example gravitational field one energy level of the atom acquires a phase shift with respect to the other level [5]. This phase shift may be detected with the help of the Ramsey interferometer (Fig. 1, upper diagram). If we have instead of this a quantum-correlated (entangled) state of two (Fig. 1, middle diagram) or many (Fig. 1, lower diagram) atoms, then the phase shift is twice or many times greater. Therefore, the sensitivity of the interferometer is increased.

Notice that an analogous proposal for the Ramsey spectrometer instead of Ramsey interferometer, which has been put forward in [6], fails. In the case of a spectrometer the deviation of the frequency of the Rabi pulse from the resonance frequency of the atom is measured. In [6] it was suggested to improve the sensitivity of the spectrometer making use of entangled atoms. However just in this case the idea does not work. The reason is that the

\footnotetext{
${ }^{1}$ The reason for the phase shift is that the path the atom is traveling along depends on its state.
} 

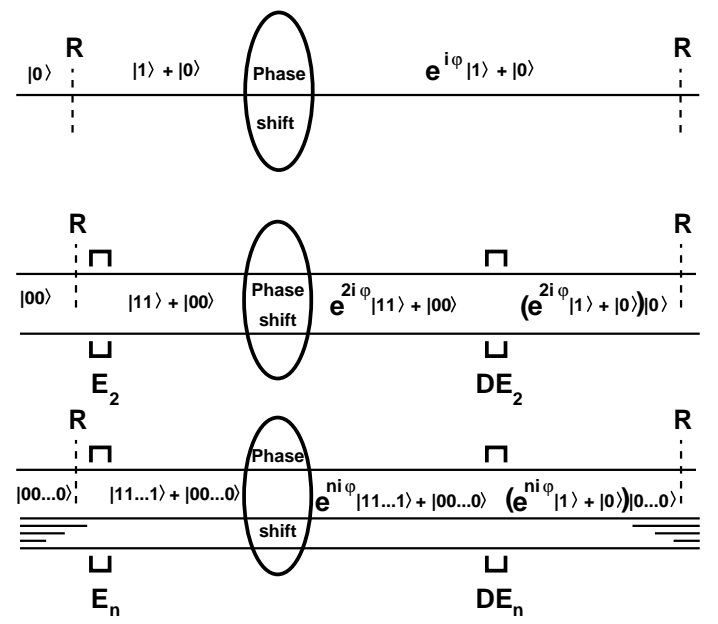

Figure 1: Ramsey interferometer with one atom (upper diagram), two entangled atoms (middle) and $n$ entangled atoms (lower). Rabi pulses $\mathrm{R}$ are applied only to the first atom, but the phase shift is determined by state differences of all entangled atoms. Device $\mathrm{E}_{n}$ realizes an entanglement of the atoms while $\mathrm{DE}_{n}$ does disentangle them. The probability for the first particle to transit from the initial state $|0\rangle$ to the final state $|1\rangle$ depends on the total phase shift which is proportional to the number of entangled atoms. The sensitivity of the interferometer to small phase shifts is thus increased by the factor $n^{2}$.

Ramsey interference in the spectrometer is owing to the phase difference of the two Rabi pulses which is determined by the flying time of the atom between the two Rabi pulses. In this case the phase shift does not depend on the number of the entangled particles (Eqs. $(5,6,8)$ and therefore most of the rest formulas in [6] contain mistakes).

\subsection{Scheme of an interferometer with entanglement}

Contrary to the case of the spectrometer, the sensitivity of the interferometer will be actually improved if the interfering atoms are entangled. A concrete scheme of the interferometer with two entangled atoms may be worked out in the following way.

The role of entanglement is easily seen from the comparison of the two diagrams of Fig. 2. The experimental setup is such that atoms in the states $|0\rangle,|1\rangle$ move in a gravitational field along one of two paths depending on the state of the given atom. This is achieved by the usual method [5], i.e. with 

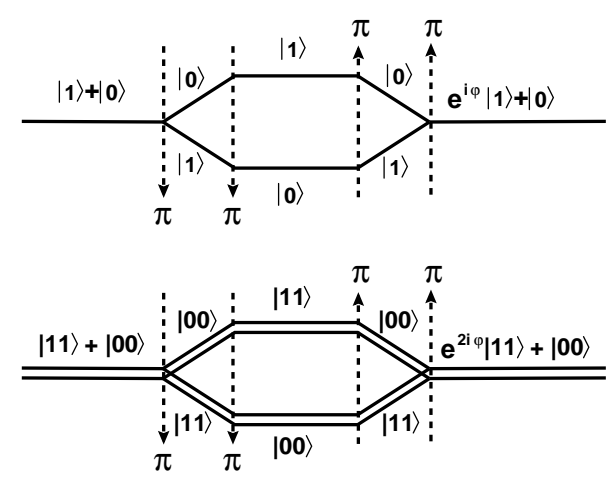

Figure 2: Atom interferometer without (upper diagram) and with (down) entanglement.

the help of $\pi$-pulses of resonance radiation in the form of running waves (the direction of the running wave is pointed out by the arrow). Acting on the ground state $|0\rangle$ this radiation causes the atom to absorb one photon. As a result, the ground state converts into the excited state $|1\rangle$. The transversal linear momentum of the atom, initially null, becomes equal to the linear momentum $k$ of the absorbed photon (directed along the direction of the running wave). On the contrary, the atom which is initially in the state $|1\rangle$ issues the same photon and therefore transits into the state $|0\rangle$ with the linear momentum $-k$. Thus, during the next period of time, the direction of motion of the atom depends on its state as it is drawn in Fig. 2. Then, after the action of the second $\pi$-pulse (with the same direction of running wave), the atoms return to the initial states with the initial null transversal linear momentum. Now each atom is moving along one of two parallel lines depending on its state. Then the pair of $\pi$-pulses with the opposite direction of running waves brings the atom together again as is shown in Fig. 2 .

Let the passage of the states $|0\rangle,|1\rangle$ along different paths in a gravitational field (as in Fig. 2) give the phase factor $\exp (i \varphi)$ only for the state $|1\rangle$. Then the 2-atom states $|11\rangle=|1\rangle|1\rangle,|00\rangle=|0\rangle|0\rangle$, when passing through the same device, will differ by the factor $\exp (2 i \varphi)$ (Fig. 2, lower diagram). The $n$-atom state $|11 \ldots 1\rangle$ will acquire the factor $\exp (n i \varphi)$ as compared to the state $|00 \ldots 0\rangle$. Therefore, the phase shift will be $n$ times greater for $n$ entangled atoms than in the case of not-entangled atoms. The sensitivity to the phase shift will be improved correspondingly.

We have still to point out a possible construction of devices $\mathrm{E}_{n}$ and $\mathrm{DE}_{n}$ of Fig. 1. In other words, we have to explain how the initial entangled state may be formed and how the final entangled state is converted to the 
disentangled one so that the phase shift may be observed experimentally. To this end, consider a scheme which realizes this task in the case $n=2$. The entanglement and disentanglement of atoms may be achieved by passing them through the resonance microcavities as it is shown in Fig. 3. The central

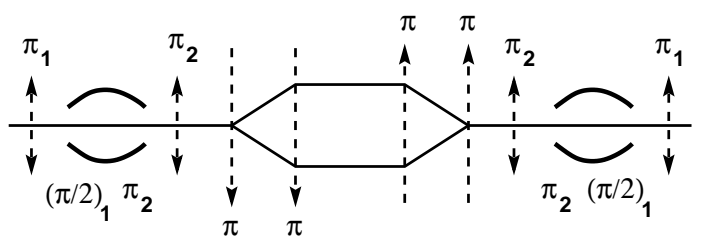

Figure 3: Scheme of an interferometer with two entangled atoms

part of the figure is identical to the lower diagram of Fig. 2. The first (left) part of Fig. 3 (device $\mathrm{E}_{2}$ ) realizes the entanglement of the atoms and the last (right) part (device $\mathrm{DE}_{2}$ ) again disentangles them.

Two atoms entering the device $\mathrm{E}_{2}$ are in the ground state $|0\rangle$ so that the 2-atom state is $|00\rangle$. Then the first atom is subject to the $\pi$-pulse of the resonance standing-wave radiation (in Fig. 3 this is denoted by $\pi_{1}$ ). This gives state $|10\rangle$. Then the first atom is flying through a microcavity during the time corresponding to the $\pi / 2$-pulse (in the figure this is denoted by $\left.(\pi / 2)_{1}\right)$. The initial state of the cavity is vacuum $|0\rangle_{\gamma}$ so that the initial state of both atoms and the microcavity is $|1\rangle_{1}|0\rangle_{\gamma}|0\rangle_{2}$. After the cavity is passed by the first atom, this state converts into the superposition $\left(|1\rangle_{1}|0\rangle_{\gamma}-|0\rangle_{1}|1\rangle_{\gamma}\right)|0\rangle_{2} / \sqrt{2}$ (a superposition of the initial state and the state resulting from radiation of a photon by the first atom into the cavity). After this the second atom is flying through the same cavity during the time period corresponding to the $\pi$-pulse (in the figure this is denoted by $\pi_{2}$ under the cavity). This gives $\left(|1\rangle_{1}|0\rangle_{2}-|0\rangle_{1}|1\rangle_{2}\right)|0\rangle_{\gamma} / \sqrt{2}$, thus the cavity is finally in the vacuum state as it has been initially. At last, the second atom is subject to the $\pi$-pulse of resonance radiation. This results in the state $\left(|1\rangle_{1}|1\rangle_{2}+|0\rangle_{1}|0\rangle_{2}\right) / \sqrt{2}$ of the atoms. Thus the entangled state $(|00\rangle+|11\rangle) / \sqrt{2}$ is formed as the output of $\mathrm{E}_{2}$.

The action of the disentangling device $\mathrm{DE}_{2}$ is analogous, but with the opposite order of all operations. Notice that the second atom has to pass the second microcavity (included in $\mathrm{DE}_{2}$ ) before the first one does so. This may be provided by a greater velocity of the second atom on its way from the first cavity to the second one.

If there is no phase shift between the states $|11\rangle$ and $|00\rangle$ traveling along different paths, then the state $|11\rangle+|00\rangle$ entering the device $\mathrm{DE}_{2}$ is converted 
into $|00\rangle$. If however the state $|11\rangle$ obtains some phase shift $2 \varphi$ during its travel in the gravitational field so that the state entering $\mathrm{DE}_{2}$ is $e^{2 i \varphi}|11\rangle+|00\rangle$ then the output state is a mixture of $|00\rangle$ and $|10\rangle$. The probability that the state $|10\rangle$ will be detected at the output is equal to

$$
\operatorname{Prob}(|10\rangle)=\frac{1}{2}(1-\cos 2 \varphi)
$$

which is equal to $\varphi^{2}$ for small $\varphi$. For comparison, in the interferometer using no entanglement (as in the upper diagram of Fig. 2) the probability to discover the phase shift $\varphi$ would be equal to $(1-\cos \varphi) / 2$ which is equal to $\varphi^{2} / 4$ for small $\varphi$.

Thus, entanglement of two atoms increases the probability to observe the phase shift by the factor 4 . If $n$ atoms are entangled, the probability will be $n^{2}$ times greater than it is in the scheme without entanglement. An experimental setup of the type presented in Fig. 3 is feasible with the present technology.

\section{ACKNOWLEDGEMENT}

The work was supported in part by the Deutsche Forschungsgemeinschaft, grant 436 RUS 17/12/00.

\section{References}

[1] A.Barenco and A.Ekert, J. Mod. Opt. 42, 1253 (1995); C.H.Bennett, Phys. Today 48, 24 (1995).

[2] A.Ekert, Phys. Rev. Lett. 67, 661 (1991); C.H.Bennett, Sci. Am. 267, 50 (1992).

[3] P.W.Shor, SIAM J. Comp. 26, 1484 (1997); L.K.Grover, Phys. Rev. Lett. 79, 325 (1997).

[4] H.-H. von Borzeszkowski and M.B. Mensky, Phys. Lett. A 269, 197 (2000).

[5] B.Mashhoon, Phys. Rev. Lett. 61, 2639 (1988); Ch.J.Borde, Phys. Lett. A 140, 10-12 (1989); J. Audretsch and K.-P. Marzlin, Phys.Rev. A50 (1994) 2080.

[6] S.F.Huelga et al., Phys. Rev. Lett. 79, 3865 (1997) quant-ph/9707014 